\title{
ON THE BOHR TRANSFORM OF ALMOST-PERIODIC SOLUTIONS FOR SOME DIFFERENTIAL EQUATIONS IN ABSTRACT SPACES
}

\author{
SAMUEL ZAIDMAN
}

(Received 11 March 2001 and in revised form 4 July 2001)

ABSTRACT. We consider abstract differential equations of the form $u^{\prime}(t)=A u(t)+f(t)$ or $u^{\prime \prime}(t)=A u(t)+f(t)$ in Banach spaces $X$, where $f(\cdot), \mathbb{R} \rightarrow X$ is almost-periodic, while $A$ is a linear operator, $\mathscr{D}(A) \subset X \rightarrow X$. If the solution $u(\cdot)$ is likewise almost-periodic, $\mathbb{R} \rightarrow X$, we establish connections between their Bohr-transforms, $\hat{u}(\lambda)$ and $\hat{f}(\lambda)$.

2000 Mathematics Subject Classification. 34C27, 34G10.

1. Introduction. If $u(\cdot), \mathbb{R} \rightarrow X$ (a Banach space) is an ultraweak almost-periodic solution of the differential equation

$$
u^{\prime}(t)=A u(t)+f(t), \quad t \in \mathbb{R} \text { (first order) }
$$

or

$$
u^{\prime \prime}(t)=A u(t)+f(t), \quad t \in \mathbb{R} \text { (second order) }
$$

with linear-not necessarily continuous-operator $A$, $\mathscr{D}(A) \subset X \rightarrow X$, and with almostperiodic "forcing" term $f(\cdot), \mathbb{R} \rightarrow X$, then the Bohr transforms

$$
\hat{f}(\lambda)=\lim _{T \rightarrow \infty} \frac{1}{T} \int_{0}^{T} e^{-i \lambda t} f(t) d t, \quad \hat{u}(\lambda)=\lim _{T \rightarrow \infty} \frac{1}{T} \int_{0}^{T} e^{-i \lambda t} u(t) d t,
$$

will both exist, for all reals $\lambda$.

It appears that it is possible to establish a typical relationship between $\hat{u}(\lambda)$ and $\hat{f}(\lambda)$ which implies also connections between the (countable) sets

$$
\Lambda_{u}=\{\lambda \in \mathbb{R}, \hat{u}(\lambda) \neq \theta\}, \quad \Lambda_{f}=\{\lambda \in \mathbb{R}, \hat{f}(\lambda) \neq \theta\},
$$

(the elements of $\Lambda_{u}, \Lambda_{f}$ are the Fourier "exponents" of $u(\cdot), f(\cdot)$, resp.).

Some "historical" notes:

(i) In [7] we have (1.2) in a Hilbert space, with $A \geq \Theta$, and the solution is in the usual sense. One obtains the equality

$$
\left(\lambda^{2}+\tilde{A}\right) \hat{u}(\lambda)=-\hat{f}(\lambda), \quad \forall \lambda \in \mathbb{R},
$$

where $\tilde{A}$ is a selfadjoint extension of $A$.

(ii) In [6] the equation is (1.1) and $f$ is continuous almost-periodic in Stepanoff $S^{P}$-sense; the operator $A$ is closed in $X$. One gets the equality

$$
(i \lambda-A) \hat{u}(\lambda)=\hat{f}(\lambda) .
$$


(iii) In [2, page 92] and [8, page 95] one has again (1.1) in the special case when $A \in \mathscr{L}(X)$.

In (i), (ii), and (iii) the solutions are regular solutions.

(iv) In $[4,5]$ one discusses (1.1) with $A$ being generator of a $C_{0}$-semigroup in the Banach space $X$. Now $u(\cdot)$ is a so-called "mild" solution over $\mathbb{R}$. It appears that $\hat{u}(\lambda) \in$ $\mathscr{D}(A)$ (even if $u(\cdot)$ does not!) and (1.6) holds again. For a detailed proof (see [11]).

The aim of the present paper is to establish similar results to those mentioned above for both (1.1) and (1.2) and for solutions taken in the "ultraweak" sense.

\section{Continuous ultraweak solutions}

2.1. We consider here the first-order equation $u^{\prime}=A u+f$ in the Banach space $X$. We refer to [10, Chapter XII] for definitions and some results.

Thus, $X$ is a $B$-space, $X^{*}$ and $X^{* *}$ its dual and second dual space. Let $A$ be a linear closed operator with dense domain in $X$, $\mathscr{D}(A)$. Assume that its dual operator $A^{*}$, is also defined on a dense domain $\mathscr{D}\left(A^{*}\right)$ in $X^{*}$. Then, the second dual operator $A^{* *}$ is well defined on the "total" set $\mathscr{D}\left(A^{* *}\right)$ in $X^{* *}$.

Consider now two continuous functions $u(\cdot), f(\cdot), \mathbb{R} \rightarrow X$ which are related by the "ultraweak" equation

$$
\int_{\mathbb{R}}\left\langle\dot{\varphi}^{*}(t)+\left(A^{*} \varphi^{*}\right)(t), u(t)\right\rangle d t=-\int_{\mathbb{R}}\left\langle\varphi^{*}(t), f(t)\right\rangle d t
$$

which must hold

$$
\begin{gathered}
\forall \varphi^{*}(\cdot) \in K_{A^{*}}(\mathbb{R})=\left\{\varphi^{*}(\cdot) \in C_{0}^{1}\left(\mathbb{R} ; X^{*}\right), \varphi^{*}(t) \in \mathscr{D}\left(A^{*}\right)\right. \\
\left.\forall t \in \mathbb{R},\left(A^{*} \varphi^{*}\right)(\cdot) \in C\left(\mathbb{R} ; X^{*}\right)\right\} .
\end{gathered}
$$

The dot $\cdot$ means $(d / d t)$-strong derivative in $X^{*}$.

Next, we need the "mollification" result: (see [10, pages 79-80]). Let $\alpha(\cdot) \in C_{0}^{1}(\mathbb{R})$. Consider the convolutions $u * \alpha, f * \alpha$ defined by equalities

$$
(u * \alpha)(t)=\int_{\mathbb{R}} u(s) \alpha(t-s) d s, \quad(f * \alpha)(t)=\int_{\mathbb{R}} f(s) \alpha(t-s) d s .
$$

It is obvious that $(u * \alpha)(\cdot) \in C^{1}(\mathbb{R} ; X)$ and $(f * \alpha)(\cdot) \in C^{1}(\mathbb{R} ; X)$ too. Furthermore, from [10, Theorem 3.1, page 80] we obtain, under our present assumptions, that

$$
\mathscr{L}(u * \alpha)(t) \in \mathscr{D}\left(A^{* *}\right) \quad \forall t \in \mathbb{R},
$$

and the equality

$$
\frac{d}{d t} \mathscr{f}(u * \alpha)(t)=A^{* *} \mathscr{g}(u * \alpha)(t)+\mathscr{g}(f * \alpha)(t)
$$

holds, in ordinary sense, for all $t \in \mathbb{R}$. (The operator, "canonical mapping" $\mathscr{f}$, from $X$ into $X^{* *}$, is defined by the equality $(\mathscr{g} x)\left(x^{*}\right)=x^{*}(x)$, for all $x^{*} \in X^{*}$, for all $x \in X$.)

Consider now the "almost-periodic situation": both $u(\cdot)$ and $f(\cdot)$ are Bohr-Bochner almost-periodic, $\mathbb{R} \rightarrow X$. 
Then, as well known (cf. [1, page 72]), the convolutions $(u * \alpha)(\cdot),(f * \alpha)(\cdot)$ are also almost-periodic, $\mathbb{R} \rightarrow X$, and, as $\mathscr{f}$ is isometric $X \rightarrow X^{* *}$, we get that the functions $\mathscr{f}(u * \alpha)(\cdot)$ and $\mathscr{f}(f * \alpha)(\cdot)$ are almost-periodic, $\mathbb{R} \rightarrow X^{* *}$. Now multiplying (2.5) by $e^{-i \lambda t}$, where $\lambda \in \mathbb{R}$, we get the equality

$$
e^{-i \lambda t} \frac{d}{d t}(\mathscr{F}(u * \alpha)(t))=e^{-i \lambda t} A^{* *} \mathscr{g}(u * \alpha)(t)+e^{-i \lambda t} \mathscr{g}(f * \alpha)(t), \quad \forall t \in \mathbb{R} .
$$

After integration between 0 and $T$ one obtains the equality

$$
\begin{aligned}
I & =\int_{0}^{T} e^{-i \lambda t} \frac{d}{d t}(\mathscr{g}(u * \alpha)(t)) d t \\
& =\int_{0}^{T} e^{-i \lambda t} A^{* *} \mathscr{g}(u * \alpha)(t) d t+\int_{0}^{T} e^{-i \lambda t} \mathscr{g}(f * \alpha)(t) d t .
\end{aligned}
$$

In the integral defining $I$ we apply integration by parts, to get

$$
I=e^{-i \lambda T} \mathscr{g}(u * \alpha)(T)-\mathscr{G}(u * \alpha)(0)+i \lambda \int_{0}^{T} e^{-i \lambda t} \mathscr{G}(u * \alpha)(t) d t .
$$

In the right-hand side of (2.7) we note the following: the second dual operator $A^{* *}$ is a closed operator; from (2.5) we derive the equality

$$
A^{* *} \mathscr{g}(u * \alpha)(t)=\frac{d}{d t} \mathscr{g}(u * \alpha)(t)-\mathscr{g}(f * \alpha)(t), \quad \forall t \in \mathbb{R},
$$

so that $A^{* *} \mathscr{g}(u * \alpha)(t) \in C\left(\mathbb{R} ; X^{* *}\right)$.

We then apply the well-known result (cf. [11, Proposition 3.1, page 162]) and obtain that

$$
\begin{gathered}
\int_{0}^{T} e^{-i \lambda t} \mathscr{G}(u * \alpha)(t) d t \in \mathscr{D}\left(A^{* *}\right), \\
A^{* *} \int_{0}^{T} e^{-i \lambda t} \mathscr{g}(u * \alpha)(t) d t=\int_{0}^{T} e^{-i \lambda t} A^{* *} \mathscr{g}(u * \alpha)(t) d t .
\end{gathered}
$$

Then we turn back to (2.7) and (2.8) to derive the equality

$$
\begin{aligned}
\frac{1}{T}\left[e^{-i \lambda T} \mathscr{g}(u * \alpha)(T)-\mathscr{G}(u * \alpha)(0)\right]+i \lambda \frac{1}{T} \int_{0}^{T} e^{-i \lambda t} \mathscr{F}(u * \alpha)(t) d t \\
=A^{* *} \frac{1}{T} \int_{0}^{T} e^{-i \lambda t} \mathscr{g}(u * \alpha)(t) d t+\frac{1}{T} \int_{0}^{T} e^{-i \lambda t} \mathscr{F}(f * \alpha)(t) d t .
\end{aligned}
$$

Next, we consider limits, as $T \rightarrow \infty$, in the right- and left-hand sides of (2.11): as the almost-periodic function $T \rightarrow \mathscr{f}(u * \alpha)(T)$ is bounded, $\mathbb{R} \rightarrow X^{* *}$, we first have

$$
\lim _{T \rightarrow \infty} \frac{1}{T}\left[e^{-i \lambda T} \mathscr{g}(u * \alpha)(T)-\mathscr{g}(u * \alpha)(0)\right]=\theta .
$$

Next, note that

$$
\begin{aligned}
& \lim _{T \rightarrow \infty} \frac{1}{T} \int_{0}^{T} e^{-i \lambda t} \mathscr{f}(u * \alpha)(t) d t \quad \text { exists and }=[\mathscr{F}(u * \alpha)]^{\Lambda}(\lambda), \\
& \lim _{T \rightarrow \infty} \frac{1}{T} \int_{0}^{T} e^{-i \lambda t} \mathscr{f}(f * \alpha)(t) d t \text { exists and }=[\mathscr{F}(f * \alpha)]^{\Lambda}(\lambda) .
\end{aligned}
$$




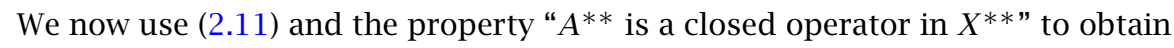

$$
\begin{gathered}
{[\mathscr{F}(u * \alpha)]^{\Lambda}(\lambda) \in \mathscr{D}\left(A^{* *}\right),} \\
i \lambda[\mathscr{F}(u * \alpha)]^{\Lambda}(\lambda)=A^{* *}[\mathscr{F}(u * \alpha)]^{\Lambda}(\lambda)+[\mathscr{F}(f * \alpha)]^{\Lambda}(\lambda) .
\end{gathered}
$$

Note also, as $\mathscr{E}$ is a continuous operator, $X \rightarrow X^{* *}$, the equalities

$$
[\mathscr{F}(u * \alpha)]^{\Lambda}(\lambda)=\mathscr{E}[(u * \alpha)]^{\Lambda}(\lambda), \quad[\mathscr{F}(f * \alpha)]^{\Lambda}(\lambda)=\mathscr{F}[f * \alpha]^{\Lambda}(\lambda) .
$$

Also, we use [1, Lemma, page 72] to derive the equalities

$$
(u * \alpha)^{\Lambda}(\lambda)=\hat{u}(\lambda) \int_{\mathbb{R}} e^{-i \lambda \sigma} \alpha(\sigma) d \sigma, \quad(f * \alpha)^{\Lambda}(\lambda)=\hat{f}(\lambda) \int_{\mathbb{R}} e^{-i \lambda \sigma} \alpha(\sigma) d \sigma .
$$

Thus, equality (2.14) becomes (in view of (2.15) and (2.16))

$$
\begin{aligned}
& i \lambda \mathscr{\hat { u }}(\lambda) \int_{\mathbb{R}} e^{-i \lambda \sigma} \alpha(\sigma) d \sigma \\
& \quad=A^{* *} \mathscr{\xi} \hat{u}(\lambda) \int_{\mathbb{R}} e^{-i \lambda \sigma} \alpha(\sigma) d \sigma+\mathscr{g} \hat{f}(\lambda) \int_{\mathbb{R}} e^{-i \lambda \sigma} \alpha(\sigma) d \sigma .
\end{aligned}
$$

Next we consider a sequence $\left\{\alpha_{p}(\cdot)\right\}$ in $C_{0}^{1}(\mathbb{R})$, where $\alpha_{p}(t)=0$ for $|t| \geq 1 / p, \alpha_{p}(t)=$ $p \alpha(p t)$, for all $t \in \mathbb{R}, p \in \mathbb{N}, \alpha(\cdot)$ being a $C_{0}^{1}(\mathbb{R})$ function which is 0 for $|t| \geq 1$, is $\geq 0$ for all $t \in \mathbb{R}$, and has $\int_{\mathbb{R}} \alpha(t) d t=1$. We note that

$$
\int_{\mathbb{R}} e^{-i \lambda \sigma} \alpha_{p}(\sigma) d \sigma=\int_{\mathbb{R}} e^{-i \lambda(s / p)} \alpha(s) d s,
$$

so that

$$
\lim _{p \rightarrow \infty} \int_{\mathbb{R}} e^{-i \lambda \sigma} \alpha_{p}(\sigma) d \sigma=\int_{\mathbb{R}} \alpha(s) d s=1
$$

Consider now equality (2.17) applied to $\alpha_{p}(\sigma)$. We have, for all $p \in \mathbb{N}$

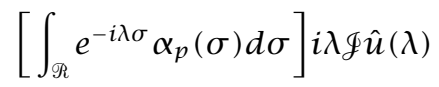

$$
\begin{aligned}
& =A^{* *} \mathscr{g} \hat{u}(\lambda) \int_{\mathbb{R}} e^{-i \lambda \sigma} \alpha_{p}(\sigma) d \sigma+\mathscr{f} \hat{f}(\lambda) \int_{\mathbb{R}} e^{-i \lambda \sigma} \alpha_{p}(\sigma) d \sigma .
\end{aligned}
$$

Again we use "closedness" of operator $A^{* *}$ and obtain that, as $p \rightarrow \infty, \mathscr{\mathscr { u }}(\lambda) \in \mathscr{D}\left(A^{* *}\right)$ and $A^{* *} \mathscr{\mathscr { u }}(\lambda)=i \lambda \mathscr{\mathscr { u }}(\lambda)-\mathscr{\mathscr { f }} \hat{f}(\lambda)$ for all $\lambda \in \mathbb{R}$, which can be written as

$$
\left(i \lambda-A^{* *}\right) \mathscr{g} \hat{u}(\lambda)=\mathscr{f} \hat{f}(\lambda), \quad \forall \lambda \in \mathbb{R} .
$$

We can summarize all of above in the following statement.

THEOREM 2.1. In the Banach space $X$ consider a linear closed operator $A$ with dense domain $\mathscr{D}(A)$, and assume that its dual operator $A^{*}$ is also densely defined in $X^{*}$. Next consider two continuous almost-periodic functions, $u(\cdot)$ and $f(\cdot)$, from $\mathbb{R}$ into $X$, related by (2.1). Then, if $\hat{u}(\lambda)$ and $\hat{f}(\lambda)$ are the Bohr transforms (1.3) and $\mathscr{f}$ is the canonical immersion of $X$ into $X^{* *}$, it follows that

$$
\mathscr{g} \hat{u}(\lambda) \in \mathscr{D}\left(A^{* *}\right) \text { and (2.21) holds true, } \quad \forall \lambda \in \mathbb{R} \text {. }
$$


Next, we are making some comments and deriving some consequences of Theorem 2.1.

(a) In (2.21), we note that if $\hat{u}(\lambda)=\theta$, then $\hat{f}(\lambda)=\theta$ (as $\mathscr{g}$ is isometric). Accordingly, if $\hat{f}(\lambda) \neq \theta$ then $\hat{u}(\lambda) \neq \theta$ which, in view of (1.4) means that

$$
\Lambda_{f} \subset \Lambda_{u}
$$

(b) Assume that $i \lambda_{0} \notin \sigma_{p}\left(A^{* *}\right)$ (the point spectrum of operator $A^{* *}$ ). Use again (2.21); if $\hat{f}\left(\lambda_{0}\right)=\theta$ then $\left(i \lambda_{0}-A^{* *}\right) \mathscr{g} \hat{u}\left(\lambda_{0}\right)=\theta$ and accordingly $\mathscr{\mathscr { L }} \hat{u}\left(\lambda_{0}\right)=\theta$ too. Thus, if $\hat{u}\left(\lambda_{0}\right) \neq \theta$ then $\hat{f}\left(\lambda_{0}\right) \neq \theta$. We can say that

$$
\lambda_{0} \in \mathbb{R}, \quad i \lambda_{0} \notin \sigma_{p}\left(A^{* *}\right), \quad \lambda_{0} \in \Lambda_{u} \Longrightarrow \lambda_{0} \in \Lambda_{f}
$$

and also that, using also (2.23),

$$
\Lambda_{u} \cap\left\{\lambda \in \mathbb{R}, i \lambda \notin \sigma_{p}\left(A^{* *}\right)\right\} \subset \Lambda_{f} \subset \Lambda_{u} .
$$

In the special case when $i \lambda \notin \sigma_{p}\left(A^{* *}\right)$ for all $\lambda \in \mathbb{R}$, we obtain from (2.25) that

$$
\Lambda_{u} \subset \Lambda_{f} \subset \Lambda_{u} \text {, hence } \Lambda_{u}=\Lambda_{f} \text {. }
$$

(c) Assume that the space $X$ is reflexive $\left(\mathscr{f}(X)=X^{* *}\right)$. From (2.21), which is also

$$
\mathscr{g}(i \lambda \hat{u}(\lambda))-A^{* *} \mathscr{g} \hat{u}(\lambda)=\mathscr{f} \hat{f}(\lambda), \quad \forall \lambda \in \mathbb{R},
$$

applying $\mathscr{g}^{-1}$, we obtain the equality

$$
i \lambda \hat{u}(\lambda)-\mathscr{F}^{-1} A^{* *} \mathscr{g} \hat{u}(\lambda)=\hat{f}(\lambda), \quad \forall \lambda \in \mathbb{R} .
$$

We know the equality $\mathscr{g}^{-1} A^{* *} \mathscr{g}=A$ (cf. [11, page 159]), hence we derive the relation, $\hat{u}(\lambda) \in \mathscr{D}(A)$ and

$$
(i \lambda-A) \hat{u}(\lambda)=\hat{f}(\lambda), \quad \forall \lambda \in \mathbb{R} .
$$

Then we may reason as in (a) and (b) above, with $A$ replacing $A^{* *}$, and obtain, in particular, that if $(i \lambda-A)^{-1}$ exists for all $\lambda \in \mathbb{R}$, then $\Lambda_{u}=\Lambda_{f}$.

2.2. In this section, we again consider ultraweak continuous In almost-periodic solutions of (1.1) and establish a certain connection between the Bohr transforms $\hat{u}(\lambda)$, $\hat{f}(\lambda)$, under somewhat different assumptions on the operator $A$; the relation between $\hat{u}(\lambda)$ and $\hat{f}(\lambda)$ is now different form (2.21). We will state (and then prove) the following theorem.

THEOREM 2.2. Let $u(\cdot), f(\cdot)$ be continuous almost-periodic functions, $\mathbb{R} \rightarrow X$ and assume that $u^{\prime}-A u=f$ in ultraweak sense. Assume also that for some complex number $\lambda_{0}$, the operator $\left(\lambda_{0} I-A\right)^{-1}$ exists and belongs to $\mathscr{L}(X)$. Then, if $\hat{u}(\lambda), \hat{f}(\lambda)$ are the Bohr transforms of $u(\cdot), f(\cdot)$, the equality

$$
(i \lambda I-A)\left(\lambda_{0} I-A\right)^{-1} \hat{u}(\lambda)=\left(\lambda_{0} I-A\right)^{-1} \hat{f}(\lambda), \quad \forall \lambda \in \mathbb{R},
$$

holds true. 
The proof here is based on the so-called "resolvent regularization" of ultraweak solutions (cf. [9, page 149]). We need in fact a slight extension of [9, Theorem, page 149], which will apply to equations $u^{\prime}-A u=f$ with $f$ not identical to zero. Thus, let us state the following.

LEMMA 2.3. In the Banach space $X$, consider the linear operator $A$ with dense domain $\mathscr{D}(A), \mathscr{D}(A) \subset X \rightarrow X$, and assume equality (2.1). Suppose furthermore that, for some $\lambda_{0} \in \mathbb{C}$, we have $\lambda_{0} \in \rho(A)$ (resolvent set of $A$ ). Let $v(t)=\left(\lambda_{0} I-A\right)^{-1} u(t), t \in \mathbb{R} \rightarrow X$. Then

$$
\begin{aligned}
& v(\cdot) \in C^{1}(\mathbb{R} ; X), \quad v(t) \in \mathscr{D}(A) \quad \forall t \in \mathbb{R}, \\
& v^{\prime}(t)-A v(t)=\left(\lambda_{0} I-A\right)^{-1} f(t), \quad t \in \mathbb{R} .
\end{aligned}
$$

Proof. We first show that the above defined-continuous function $v(\cdot)$ is ultraweak solution of (2.31).

Take any test-function $\varphi^{*}(\cdot) \in K_{A^{*}}(\mathbb{R})$. We obviously have the equality

$$
\int_{\mathbb{R}}\left\langle\frac{d}{d t} \varphi^{*}+A^{*} \varphi^{*}, v\right\rangle d t=\int_{\mathbb{R}}\left\langle\frac{d}{d t} \varphi^{*}+A^{*} \varphi^{*},\left(\lambda_{0}-A\right)^{-1} u\right\rangle d t,
$$

where $\langle$,$\rangle means the duality between X$ and $X^{*}$ (dual space to $X$ ). We next use the well-known result (cf. [3, (4.10), page 14]) to get $\lambda_{0} \in \rho\left(A^{*}\right)$ (resolvent set of operator $\left.A^{*}\right)$ and $\left[\left(\lambda_{0}-A\right)^{-1}\right]^{*}=\left(\lambda_{0}-A^{*}\right)^{-1}$. Equality (2.32) now becomes

$$
\begin{aligned}
\int_{\mathbb{R}}\left\langle\frac{d}{d t} \varphi^{*}+A^{*} \varphi^{*}, v\right\rangle d t & =\int_{\mathbb{R}}\left\langle\left(\lambda_{0}-A^{*}\right)^{-1}\left(\frac{d}{d t} \varphi^{*}+A^{*} \varphi^{*}\right), u\right\rangle d t \\
& =\int_{\mathbb{R}}\left\langle\frac{d}{d t}\left(\lambda_{0}-A^{*}\right)^{-1} \varphi^{*}+A^{*}\left(\lambda_{0}-A^{*}\right)^{-1} \varphi^{*}, u\right\rangle d t \\
& =\int_{\mathbb{R}}\left\langle\frac{d}{d t} \psi^{*}+A^{*} \psi^{*}, u\right\rangle d t
\end{aligned}
$$

where $\psi^{*}=\left(\lambda_{0}-A^{*}\right)^{-1} \varphi^{*}$ belongs (obviously) to $K_{A^{*}}(\mathbb{R})$. Next, because of (2.1), we see that

$$
\begin{aligned}
\int_{\mathbb{R}}\left\langle\frac{d}{d t} \psi^{*}+A^{*} \psi^{*}, u\right\rangle d t & =-\int_{\mathbb{R}}\left\langle\psi^{*}, f\right\rangle d t \\
& =-\int_{\mathbb{R}}\left\langle\left(\lambda_{0}-A^{*}\right)^{-1} \varphi^{*}, f\right\rangle d t \\
& =-\int_{\mathbb{R}}\left\langle\varphi^{*},\left(\lambda_{0}-A\right)^{-1} f\right\rangle d t
\end{aligned}
$$

Thus, from (2.32) and (2.34) we derive the relation

$$
\int_{\mathbb{R}}\left\langle\frac{d}{d t} \varphi^{*}+A^{*} \varphi^{*}, v\right\rangle d t=-\int_{\mathbb{R}}\left\langle\varphi^{*},\left(\lambda_{0}-A\right)^{-1} f\right\rangle d t, \quad \forall \varphi^{*} \in K_{A^{*}}(\mathbb{R})
$$

which means precisely that (2.31) holds in the ultraweak sense.

Next, note the simple [9, Lemma 2, page 150]: $v(t) \in \mathscr{D}(A)$ for all $t \in \mathbb{R}$, and $(A v)(\cdot) \in C(\mathbb{R} ; X)$; then apply [10, Proposition 2.1, page 79] to the functions $v(\cdot)$ and $\left(\lambda_{0}-A\right)^{-1} f$. (Note that, because $\lambda_{0} \in \rho(A)$ it results that $A$ is closed.) One obtains that $v(\cdot) \in C^{1}(\mathbb{R} ; X)$ and (2.31) holds true on the real line. 
We are now ready for the complete proof of Theorem 2.2.

Consider (2.31) and note that, from almost-periodicity of $u(\cdot)$ and $f(\cdot)$ the almostperiodicity of $v(\cdot)$ and $\left(\lambda_{0}-A\right)^{-1} f$ follows. Then multiply (2.31) by $e^{-i \lambda t}, \lambda \in \mathbb{R}$, to get the equality

$$
e^{-i \lambda t} v^{\prime}(t)-e^{-i \lambda t} A v(t)=e^{-i \lambda t}\left(\lambda_{0}-A\right)^{-1} f(t), \quad t \in \mathbb{R} .
$$

Then integrate between 0 and $T: \int_{0}^{T} e^{-i \lambda t} v^{\prime}(t) d t=e^{-i \lambda T} v(T)-v(0)+\int_{0}^{T} i \lambda e^{-i \lambda t} v(t) d t$ so that one gets from (2.36)

$$
\begin{array}{rl}
e^{-i \lambda T} & v(T)-v(0)+i \lambda \int_{0}^{T} e^{-i \lambda t} v(t) d t-\int_{0}^{T} A\left(e^{-i \lambda t} v(t)\right) d t \\
& =\int_{0}^{T} e^{-i \lambda t}\left(\lambda_{0}-A\right)^{-1} f(t) d t
\end{array}
$$

Also, as usual ( $A$ is a closed operator) one obtains

$$
\int_{0}^{T} e^{-i \lambda t} v(t) d t \in \mathscr{D}(A), \quad A \int_{0}^{T} e^{-i \lambda t} v(t) d t=\int_{0}^{T} A\left(e^{-i \lambda t} v(t)\right) d t .
$$

Thus, from (2.37) one gets now the equality

$$
\begin{gathered}
\frac{1}{T}\left[e^{-i \lambda T} v(T)-v(0)\right]+i \lambda \frac{1}{T} \int_{0}^{T} e^{-i \lambda t} v(t) d t-A \frac{1}{T} \int_{0}^{T} e^{-i \lambda t} v(t) d t \\
=\frac{1}{T} \int_{0}^{T} e^{-i \lambda t}\left(\lambda_{0}-A\right)^{-1} f(t) d t .
\end{gathered}
$$

As $T \rightarrow \infty$ one obtains as in (2.12), (2.13), the equality

$$
i \lambda \hat{v}(\lambda)-A \hat{v}(\lambda)=\left(\left(\lambda_{0}-A\right)^{-1} f\right)^{\Lambda}(\lambda) .
$$

On the other hand, it is immediate that $\hat{v}(\lambda)=\left(\lambda_{0}-A\right)^{-1} \hat{u}(\lambda)$, and $\left(\left(\lambda_{0}-A\right)^{-1} f\right)^{\Lambda}(\lambda)$ $=\left(\lambda_{0}-A\right)^{-1} \hat{f}(\lambda)$. Hence (2.40) is also

$$
(i \lambda)\left(\lambda_{0}-A\right)^{-1} \hat{u}(\lambda)-A\left(\lambda_{0}-A\right)^{-1} \hat{u}(\lambda)=\left(\lambda_{0}-A\right)^{-1} \hat{f}(\lambda)
$$

which is exactly (2.30).

Next, as in Section 2.2, we derive some simple consequences of (2.30).

(a) Assume $\hat{u}(\lambda)=\theta$; then $\left(\lambda_{0} I-A\right)^{-1} \hat{f}(\lambda)=\theta$ and hence, $\hat{f}(\lambda)=\theta$ too. This means again that

$$
\Lambda_{f} \subset \Lambda_{u}
$$

(b) Assume that, for some $\bar{\lambda} \in \mathbb{R}, i \bar{\lambda} \notin \sigma_{p}(A)$. If $\hat{f}(\bar{\lambda})=\theta$ then $(i \bar{\lambda}-A)$ $\left(\lambda_{0}-A\right)^{-1} \hat{u}(\bar{\lambda})=\theta$, hence $\left(\lambda_{0}-A\right)^{-1} \hat{u}(\bar{\lambda})=\theta$ which implies that $\hat{u}(\bar{\lambda})=\theta$. Thus, if $\hat{u}(\bar{\lambda}) \neq \theta$ and $i \bar{\lambda} \notin \sigma_{p}(A)$, then $\hat{f}(\lambda) \neq \theta$. This can be expressed as

$$
\Lambda_{u} \cap\left\{\lambda \in \mathbb{R}, i \lambda \notin \sigma_{p}(A)\right\} \subset \Lambda_{f} \subset \Lambda_{u} .
$$

Finally, in the special case when $i \lambda \notin \sigma_{p}(A)$ for all $\lambda \in \mathbb{R}$, one obtains

$$
\Lambda_{u}=\Lambda_{f}
$$


3. The second-order equation. In this section, we study similar problems for the second-order equation (1.2) in both ordinary and ultraweak setting.

First, we state the result for regular solutions.

TheOrem 3.1. Let $X$ be a $B$-space, and $A, \mathscr{D}(A) \subset X \rightarrow X$ a linear closable operator. Let $u(\cdot)$ and $f(\cdot)$ be continuous almost-periodic functions, $\mathbb{R} \rightarrow X$, let also $u(\cdot) \in C^{2}(\mathbb{R} ; X), u(t) \in \mathscr{D}(A)$ for all $t \in \mathbb{R}$, and (1.2) holds true.

Finally, assume that $u^{\prime}(\cdot)$ is a bounded function, $\mathbb{R} \rightarrow X$. It follows that

$$
\hat{u}(\lambda) \in \mathscr{D}(\tilde{A}), \quad \forall \lambda \in \mathbb{R}, \quad\left(\lambda^{2} I+\tilde{A}\right) \hat{u}(\lambda)=-\hat{f}(\lambda), \quad \forall \lambda \in \mathbb{R},
$$

hold true.

(Here $\tilde{A}$ is a closed extension of $A$.)

Proof. We start with the equality $u^{\prime \prime}(t)=A u(t)+f(t), t \in \mathbb{R}$. As $A \subset \tilde{A}$, we have also $u^{\prime \prime}(t)=\tilde{A} u(t)+f(t), t \in \mathbb{R}$. Take $\lambda \in \mathbb{R}$; we obtain immediately the equality (for all $T>0)$

$$
\int_{0}^{T} e^{-i \lambda t} u^{\prime \prime}(t) d t=\int_{0}^{T} e^{-i \lambda t} \tilde{A} u(t) d t+\int_{0}^{T} e^{-i \lambda t} f(t) d t, \quad \forall \lambda \in \mathbb{R} .
$$

Using partial integration we get

$$
\begin{aligned}
\frac{1}{T} \int_{0}^{T} e^{-i \lambda t} u^{\prime \prime}(t) d t= & \frac{1}{T} e^{-i \lambda T} u^{\prime}(T)-\frac{u^{\prime}(0)}{T}+\frac{i \lambda e^{-i \lambda T} u(T)}{T} \\
& -\frac{i \lambda u(0)}{T}-\lambda^{2} \frac{1}{T} \int_{0}^{T} e^{-i \lambda t} u(t) d t
\end{aligned}
$$

As $T \rightarrow \infty$ the right-hand side in (3.3) is convergent to $-\lambda^{2} \hat{u}(\lambda)$, for all $\lambda \in \mathbb{R}$. On the other hand, (3.2) writes itself as

$$
\frac{1}{T} \int_{0}^{T} e^{-i \lambda t} \tilde{A} u(t) d t=\frac{1}{T} e^{-i \lambda t} u^{\prime \prime}(t) d t-\frac{1}{T} \int_{0}^{T} e^{-i \lambda t} f(t) d t
$$

As $\tilde{A}$ is closed, $u(t) \in \mathscr{D}(\tilde{A})$ for all $t \in \mathbb{R}, \tilde{A} u(t)=u^{\prime \prime}(t)-f(t) \in C(\mathbb{R} ; X)$, it follows as usual that

$$
\begin{gathered}
\frac{1}{T} \int_{0}^{T} e^{-i \lambda t} u(t) d t \in \mathscr{D}(\tilde{A}) \\
\tilde{A} \frac{1}{T} \int_{0}^{T} e^{-i \lambda t} u(t) d t=\frac{1}{T} \int_{0}^{T} e^{-i \lambda t} u^{\prime \prime}(t) d t-\frac{1}{T} \int_{0}^{T} e^{-i \lambda t} f(t) d t
\end{gathered}
$$

As $T \rightarrow \infty$, the right-hand side of (3.5) is convergent to $-\lambda^{2} \hat{u}(\lambda)-\hat{f}(\lambda)$, for all $\lambda \in \mathbb{R}$. Also, $(1 / T) \int_{0}^{T} e^{-i \lambda t} u(t) d t \rightarrow \hat{u}(\lambda)$, for all $\lambda \in \mathbb{R}$.

Again we use the property, " $\tilde{A}$ is closed" and infer that $\hat{u}(\lambda) \in \mathscr{D}(\tilde{A})$ for all $\lambda \in \mathbb{R}$, $\tilde{A} \hat{u}(\lambda)=-\lambda^{2} \hat{u}(\lambda)-\hat{f}(\lambda)$, for all $\lambda \in \mathbb{R}$, that is, (3.1).

REMARK 3.2. Theorem 3.1 extends the statement of [7, Theorem]; the proof is the same too. 
We next derive corollaries of (3.1), connecting the sets $\Lambda_{u}$ and $\Lambda_{f}$.

(a) If $\hat{u}(\lambda)=\theta$ then $\hat{f}(\lambda)=\theta$; therefore

$$
\Lambda_{f} \subset \Lambda_{u}
$$

(b) Assume that $\lambda_{0} \in \mathbb{R}$ is such that $\lambda_{0}^{2}$ is not an eigenvalue of operator $-\tilde{A}$. Then, from (3.1) again, it follows that $\hat{f}\left(\lambda_{0}\right)=\theta \Rightarrow \hat{u}\left(\lambda_{0}\right)=\theta$, or $\hat{u}\left(\lambda_{0}\right) \neq \theta \Rightarrow \hat{f}\left(\lambda_{0}\right) \neq \theta$. We can say that

$$
\Lambda_{u} \cap\left\{\lambda \in \mathbb{R}, \lambda^{2} \notin \sigma_{p}(-\tilde{A})\right\} \subset \Lambda_{f} \subset \Lambda_{u} .
$$

Next we will handle-for a similar result-the class of "ultraweak solutions." Therefore, let again $X$ be a $B$-space, and $A, \mathscr{D}(A) \subset X \rightarrow X$ be a linear operator with dense domain $\mathscr{D}(A)$. Then (cf. [11, pages 72-73]) its dual operator $A^{*}$ is a linear closed operator with domain $\mathscr{D}\left(A^{*}\right) \subset X^{*}$-the dual space of $X$.

Denote with $K_{A^{*}}^{2}(\mathbb{R})$ the (linear) set of functions $\varphi^{*}(\cdot) \in C_{0}^{2}\left(\mathbb{R} ; X^{*}\right), \varphi^{*}(t) \in \mathscr{D}\left(A^{*}\right)$ for all $t \in \mathbb{R},\left(A^{*} \varphi^{*}\right)(\cdot) \in C\left(\mathbb{R} ; X^{*}\right)$.

Next, consider two continuous functions $u(\cdot)$ and $f(\cdot), \mathbb{R} \rightarrow X$, related by the ultraweak equation

$$
\int_{\mathbb{R}}\left\langle\ddot{\varphi}^{*}-A^{*} \varphi^{*}(t), u(t)\right\rangle d t=\int_{\mathbb{R}}\left\langle\varphi^{*}(t), f(t)\right\rangle d t, \quad \forall \varphi^{*}(\cdot) \in K_{A^{*}}^{2}(\mathbb{R}) .
$$

Assume furthermore that

$A$ is a closed operator; the domain $\mathscr{D}\left(A^{*}\right)$ is dense in $X^{*}$.

We effectuate "mollification" of $u(\cdot)$-in a similar way to [10, pages 79-80].

Consider a scalar-valued function $\alpha_{\varepsilon}(\cdot) \in C_{0}^{2}(\mathbb{R})$, such that $\alpha_{\varepsilon}(t)=0$ for $|t| \geq \varepsilon$, and then the convolution

$$
\left(u * \alpha_{\varepsilon}\right)(t)=\int_{\mathbb{R}} u(\tau) \alpha_{\varepsilon}(t-\tau) d \tau=\int_{t-\varepsilon}^{t+\varepsilon} u(\tau) \alpha_{\varepsilon}(t-\tau) d \tau .
$$

We see that $\left(u * \alpha_{\varepsilon}\right)(\cdot) \in C^{2}(\mathbb{R} ; X)$ and that the equality

$$
\left(u * \alpha_{\varepsilon}\right)^{\prime \prime}(t)=\int_{\mathbb{R}} u(\tau) \alpha_{\varepsilon}^{\prime \prime}(t-\tau) d \tau, \quad \forall t \in \mathbb{R},
$$

holds true. We prove now the following lemma.

LEMMA 3.3. Under the above assumptions, $\mathscr{E}\left(u * \alpha_{\varepsilon}\right)(t) \in \mathscr{D}\left(A^{* *}\right)$ for all $t \in \mathbb{R}$ and the equality

$$
\frac{d^{2}}{d t^{2}} \mathscr{g}\left(u * \alpha_{\varepsilon}\right)(t)=A^{* *} \mathscr{g}\left(u * \alpha_{\varepsilon}\right)(t)+\mathscr{g}\left(f * \alpha_{\varepsilon}\right)(t), \quad \forall t \in \mathbb{R},
$$

holds true ( $\&$ is the canonical mapping, $X \rightarrow X^{* *}$ ).

Proof. For any $t \in \mathbb{R}$, consider the functions $\varphi_{t, \varepsilon}^{*}(\tau)=\alpha_{\varepsilon}(t-\tau) \phi^{*}$, where $\phi^{*} \in$ $\mathscr{D}\left(A^{*}\right)$ (they all belong to $K_{A^{*}}^{2}(\mathbb{R})$ - as readily seen).

We introduce these functions in (3.8); note that $\left(d^{2} / d \tau^{2}\right) \varphi_{t, \varepsilon}^{*}(\tau)=\alpha_{\varepsilon}^{\prime \prime}(t-\tau) \phi^{*}$ and obtain accordingly the relation

$$
\int_{\mathbb{R}}\left\langle\alpha_{\varepsilon}^{\prime \prime}(t-\tau) \phi^{*}-\alpha_{\varepsilon}(t-\tau) A^{*} \phi^{*}, u(\tau)\right\rangle d \tau=\int_{\mathbb{R}}\left\langle\alpha_{\varepsilon}(t-\tau) \phi^{*}, f(\tau)\right\rangle d \tau
$$


or also

$$
\begin{aligned}
\left\langle A^{*} \phi^{*}, \int_{\mathbb{R}} \alpha_{\varepsilon}(t-\tau) u(\tau) d \tau\right\rangle= & -\left\langle\phi^{*}, \int_{\mathbb{R}} \alpha_{\varepsilon}(t-\tau) f(\tau) d \tau\right\rangle \\
& +\left\langle\phi^{*}, \int_{\mathbb{R}} \alpha_{\varepsilon}^{\prime \prime}(t-\tau) u(\tau) d \tau\right\rangle \quad \forall \phi^{*} \in \mathscr{D}\left(A^{*}\right) .
\end{aligned}
$$

This equality can also be written as follows (using the imbedding $X \stackrel{\mathscr{G}}{\rightarrow} X^{* *}$ )

$$
\left\langle\mathscr{F}\left(u * \alpha_{\varepsilon}\right)(t), A^{*} \phi^{*}\right\rangle=\left\langle\mathscr{F}\left[\left(u * \alpha_{\varepsilon}\right)^{\prime \prime}(t)-\left(f * \alpha_{\varepsilon}\right)(t)\right], \phi^{*}\right\rangle \quad \forall \phi^{*} \in \mathscr{D}\left(A^{*}\right)
$$

which in turn implies $\mathscr{g}\left(u * \alpha_{\varepsilon}\right)(t) \in \mathscr{D}\left(A^{* *}\right)$ and then

$$
A^{* *}\left(\mathscr{g}\left(u * \alpha_{\varepsilon}\right)(t)\right)=\mathscr{F}\left[\left(u * \alpha_{\varepsilon}\right)^{\prime \prime}(t)-\left(f * \alpha_{\varepsilon}\right)(t)\right] \quad \forall t \in \mathbb{R} .
$$

Finally, $\mathscr{f}\left(u * \alpha_{\varepsilon}\right)^{\prime \prime}(t)=\left(d^{2} / d t^{2}\right) \mathscr{g}\left(u * \alpha_{\varepsilon}\right)(t)$; hence from (3.16) we derive

$$
\frac{d^{2}}{d t^{2}} \mathscr{F}\left(u * \alpha_{\varepsilon}\right)(t)=A^{* *}\left(\mathscr{g}\left(u * \alpha_{\varepsilon}\right)(t)\right)+\mathscr{g}\left(f * \alpha_{\varepsilon}\right)(t) \quad \forall t \in \mathbb{R} .
$$

Which proves Lemma 3.3.

We are now ready for consideration of an "extension" of Theorem 3.1 to the case of ultraweak solutions. We can state in fact the following theorem.

THEOREM 3.4. In the Banach space $X$ consider a linear closed operator $A$ with dense domain $\mathscr{D}(A)$ and assume that its dual operator $A^{*}$ is also densely defined in $X^{*}$. Then consider two continuous almost-periodic functions, $\mathbb{R} \rightarrow X$, denoted with $u(\cdot), f(\cdot)$, related by (3.8). Then, if $\hat{u}(\lambda), \hat{f}(\lambda)$ are the Bohr transforms of $u(\cdot), f(\cdot)$, it follows that $\mathscr{F} \hat{u}(\lambda) \in \mathscr{D}\left(A^{* *}\right)$ and the equality

$$
\left(\lambda^{2} I+A^{* *}\right) \mathscr{g} \hat{u}(\lambda)=-\mathscr{f} \hat{f}(\lambda) \quad \forall \lambda \in \mathbb{R}
$$

is satisfied.

The proof goes on similar lines to the proof of Theorem 2.1.

Note first that the functions: $\mathscr{f}\left(u * \alpha_{\varepsilon}\right)(\cdot), \mathscr{F}\left(f * \alpha_{\varepsilon}\right)(\cdot)$, are almost-periodic, $\mathbb{R} \rightarrow$ $X^{* *}$. Next we multiply (3.17) by $e^{-i \lambda t}$ and integrate over $[0, T], T>0$. We obtain

$$
\begin{aligned}
& \int_{0}^{T} e^{-i \lambda t} \frac{d^{2}}{d t^{2}}\left(\mathscr{F}\left(u * \alpha_{\varepsilon}\right)(t)\right) d t \\
& \quad=\int_{0}^{T} e^{-i \lambda t} A^{* *} \mathscr{g}\left(u * \alpha_{\varepsilon}\right)(t) d t+\int_{0}^{T} e^{-i \lambda t} \mathscr{F}\left(f * \alpha_{\varepsilon}\right)(t) d t .
\end{aligned}
$$

As previously, the left-hand side here is $e^{-i \lambda T} \mathscr{g}\left(u * \alpha_{\varepsilon}\right)^{\prime}(T)-\mathscr{g}\left(u * \alpha_{\varepsilon}\right)^{\prime}(0)+i \lambda \mathscr{g}(u *$ $\left.\alpha_{\varepsilon}\right)(0)-\lambda^{2} \int_{0}^{T} e^{-i \lambda t} g\left(u * \alpha_{\varepsilon}\right)(t) d t$.

If we divide this left-hand side by $T$ we obtain

$$
\begin{aligned}
\frac{1}{T} \int_{0}^{T} e^{-i \lambda t} & \frac{d^{2}}{d t^{2}}\left(\mathscr{F}\left(u * \alpha_{\varepsilon}\right)(t)\right) d t \\
= & e^{-i \lambda t} \frac{\mathscr{\mathscr { g }}\left(u * \alpha_{\varepsilon}\right)^{\prime}(T)}{T}-\frac{1}{T} \mathscr{g}\left(u * \alpha_{\varepsilon}\right)^{\prime}(0) \\
& +i \lambda \frac{\mathscr{g}\left(u * \alpha_{\varepsilon}\right)(0)}{T}-\lambda^{2} \frac{1}{T} \int_{0}^{T} e^{-i \lambda t} \mathscr{g}\left(u * \alpha_{\varepsilon}\right)(t) d t
\end{aligned}
$$


In order to proceed further we now need the following proposition.

Proposition 3.5. The function $\mathscr{f}\left(u * \alpha_{\varepsilon}\right)^{\prime}(\cdot)$ is bounded over $\mathbb{R}$.

We have in fact (from (3.10)) $\mathscr{g}\left(u * \alpha_{\varepsilon}\right)^{\prime}(t)=\int_{\mathbb{R}}(\mathscr{F} u)(\tau) \alpha_{\varepsilon}^{\prime}(t-\tau) d \tau$, hence

$$
\begin{aligned}
\left\|\mathscr{F}\left(u * \alpha_{\varepsilon}\right)^{\prime}(t)\right\| & \leq \sup _{\mathbb{R}}\|(\mathscr{} u)(\tau)\| \int_{\mathbb{R}}\left|\alpha_{\varepsilon}^{\prime}(t-\tau)\right| d \tau \\
& =c \int_{\mathbb{R}}\left|\alpha_{\varepsilon}^{\prime}(s)\right| d s=c_{1, \varepsilon} \quad \forall t \in \mathbb{R} .
\end{aligned}
$$

Thus, from (3.20) we get, letting $T \rightarrow \infty$, the relation

$$
\lim _{T \rightarrow \infty} \frac{1}{T} \int_{0}^{T} e^{-i \lambda t} \frac{d^{2}}{d t^{2}}\left(\mathscr{F}\left(u * \alpha_{\varepsilon}\right)(t)\right) d t=-\lambda^{2}\left(\mathscr{F}\left(u * \alpha_{\varepsilon}\right)\right)^{\Lambda}(\lambda) .
$$

Next, we divide by $T$ the right-hand side of (3.19). We obtain accordingly the equality

$$
\begin{gathered}
\frac{1}{T} \int_{0}^{T} e^{-i \lambda t} A^{* *} \mathscr{F}\left(u * \alpha_{\varepsilon}\right)(t) d t+\frac{1}{T} \int_{0}^{T} e^{-i \lambda t} \mathscr{g}\left(f * \alpha_{\varepsilon}\right)(t) d t \\
=\frac{1}{T} \int_{0}^{T} e^{-i \lambda t} \frac{d^{2}}{d t^{2}} \mathscr{F}\left(u * \alpha_{\varepsilon}\right)(t) d t
\end{gathered}
$$

hence

$$
\lim _{T \rightarrow \infty} \frac{1}{T} \int_{0}^{T} e^{-i \lambda t} A^{* *} \mathscr{g}\left(u * \alpha_{\varepsilon}\right)(t) d t=-\mathscr{g}\left(f * \alpha_{\varepsilon}\right)^{\Lambda}(\lambda)-\lambda^{2} \mathscr{g}\left(u * \alpha_{\varepsilon}\right)^{\Lambda}(\lambda) .
$$

In the left-hand side note that $A^{* *}$ is closed, $A^{* *} \mathscr{g}\left(u * \alpha_{\varepsilon}\right)(t)=\left(d^{2} / d t^{2}\right) \mathscr{g}\left(u * \alpha_{\varepsilon}\right)(t)-$ $\mathscr{g}\left(f * \alpha_{\varepsilon}\right)(t)$-from (3.17)-hence $A^{* *} g\left(u * \alpha_{\varepsilon}\right)(t)$ is continuous function (belongs to $\left.C\left(\mathbb{R} ; X^{* *}\right)\right)$. Thus, as usual, we infer that

$$
\begin{gathered}
\int_{0}^{T} e^{-i \lambda t} \mathscr{g}\left(u * \alpha_{\varepsilon}\right)(t) d t \in \mathscr{D}\left(A^{* *}\right), \\
A^{* *} \int_{0}^{T} e^{-i \lambda t} \mathscr{F}\left(u * \alpha_{\varepsilon}\right)(t) d t=\int_{0}^{T} e^{-i \lambda t} A^{* *} \mathscr{F}\left(u * \alpha_{\varepsilon}\right)(t) d t .
\end{gathered}
$$

Again, from (3.24) it then follows that

$$
\lim _{T \rightarrow \infty} A^{* *} \frac{1}{T} \int_{0}^{T} e^{-i \lambda t} \mathscr{F}\left(u * \alpha_{\varepsilon}\right)(t) d t \text { exists and }=-\mathscr{g}\left(f * \alpha_{\varepsilon}\right)^{\Lambda}(\lambda)-\lambda^{2} \mathscr{g}\left(u * \alpha_{\varepsilon}\right)^{\Lambda}(\lambda) .
$$

Now, from (3.24) and (3.26), as $A^{* *}$ is closed, we obtain

$$
\begin{gathered}
\mathscr{g}\left(u * \alpha_{\varepsilon}\right)^{\Lambda}(\lambda) \in \mathscr{D}\left(A^{* *}\right) \quad(\forall \lambda \in \mathbb{R}), \\
A^{* *} \mathscr{g}\left(u * \alpha_{\varepsilon}\right)^{\Lambda}(\lambda)=-\mathscr{g}\left(f * \alpha_{\varepsilon}\right)^{\Lambda}(\lambda)-\lambda^{2} \mathscr{g}\left(u * \alpha_{\varepsilon}\right)^{\Lambda}(\lambda) .
\end{gathered}
$$

we use [1, Lemma, page 72] to derive equalities

$$
\begin{aligned}
& \mathscr{F}\left(u * \alpha_{\varepsilon}\right)^{\Lambda}(\lambda)=\mathscr{g} \hat{u}(\lambda) \int_{\mathbb{R}} e^{-i \lambda \sigma} \alpha_{\varepsilon}(\sigma) d \sigma, \\
& \mathscr{J}\left(f * \alpha_{\varepsilon}\right)^{\Lambda}(\lambda)=\mathscr{g} \hat{f}(\lambda) \int_{\mathbb{R}} e^{-i \lambda \sigma} \alpha_{\varepsilon}(\sigma) d \sigma,
\end{aligned}
$$


and accordingly one gets

$$
\begin{aligned}
& A^{* *} \mathscr{\xi} \hat{u}(\lambda) \int_{\mathbb{R}} e^{-i \lambda \sigma} \alpha_{\varepsilon}(\sigma) d \sigma \\
& \quad=-\Phi \hat{f}(\lambda) \int_{\mathbb{R}} e^{-i \lambda \sigma} \alpha_{\varepsilon}(\sigma) d \sigma-\lambda^{2} \mathscr{\xi} \hat{u}(\lambda) \int_{\mathbb{R}} e^{-i \lambda \sigma} \alpha_{\varepsilon}(\sigma) d \sigma \quad \forall \lambda \in \mathbb{R} .
\end{aligned}
$$

Consider at this point a sequence $\left\{\alpha_{p}(\cdot)\right\}$ in $C_{0}^{2}(\mathbb{R})$, where $\alpha_{p}(t)=p \alpha(p t)$ for all $p \in \mathbb{N}, \alpha(\cdot)$ is a function in $C_{0}^{2}(\mathbb{R})$ which is 0 for $|t| \geq 1$, is greater than or equal to 0 for all $t \in \mathbb{R}$, has integral over $\mathbb{R}=1$. Note therefore that

$$
\begin{aligned}
\int_{\mathbb{R}} e^{-i \lambda \sigma} \alpha_{p}(\sigma) d \sigma & =\int_{\mathbb{R}} e^{-i \lambda \sigma} p \alpha(p \sigma) d \sigma \\
& =\int_{\mathbb{R}} e^{-i \lambda(s / p)} \alpha(s) d s \text { which } \longrightarrow 1 \text { as } p \longrightarrow \infty .
\end{aligned}
$$

Consider now (3.29) for $\alpha_{\varepsilon}(\sigma)=\alpha_{p}(\sigma), p \in \mathbb{N}$. As $p \rightarrow \infty$, we have

$$
\begin{aligned}
& \mathscr{\xi} \hat{u}(\lambda) \int_{\mathbb{R}} e^{-i \lambda \sigma} \alpha_{p}(\sigma) d \sigma \rightarrow \oiint \hat{u}(\lambda), \\
& A^{* *} \mathscr{g} \hat{u}(\lambda) \int_{\mathbb{R}} e^{-i \lambda \sigma} \alpha_{p}(\sigma) d \sigma \rightarrow-\mathscr{f} \hat{f}(\lambda)-\lambda^{2} \mathscr{\mathscr { u }}(\lambda) \quad \forall \lambda \in \mathbb{R} .
\end{aligned}
$$

This entails (again!), that $\mathscr{f} \hat{u}(\lambda) \in \mathscr{D}\left(A^{* *}\right)$ and

$$
A^{* *} \mathscr{g} \hat{u}(\lambda)=-\mathscr{f} \hat{f}(\lambda)-\lambda^{2} \mathscr{g} \hat{u}(\lambda) \quad \forall \lambda \in \mathbb{R}
$$

which is precisely (3.18).

REMARK 3.6. The convolution method which has been used in the proof of Theorem 3.4 can be used to get an extension of Theorem 3.1. Precisely, we can eliminate the assumption

$$
u^{\prime}(\cdot) \text { is a bounded function, } \mathbb{R} \rightarrow X .
$$

The details are given below.

We start with the equality

$$
u^{\prime \prime}(t)=\tilde{A} u(t)+f(t), \quad t \in \mathbb{R} .
$$

Take $\alpha_{\varepsilon}(\cdot) \in C_{0}^{2}(\mathbb{R}), \alpha_{\varepsilon}(t)=0$ for $|t| \geq \varepsilon$, and let

$$
u_{\varepsilon}(t)=\left(u * \alpha_{\varepsilon}\right)(t)=\int_{\mathbb{R}} u(\tau) \alpha_{\varepsilon}(t-\tau) d \tau=\int_{\mathbb{R}} u(t-s) \alpha_{\varepsilon}(s) d s .
$$

We have obviously, $u_{\varepsilon}^{\prime \prime}(t)=\int_{\mathbb{R}} u^{\prime \prime}(t-s) \alpha_{\varepsilon}(s) d s$.

Also $u(t-s) \in \mathscr{D}(\tilde{A})$ and $\tilde{A} u(t-s)=u^{\prime \prime}(t-s)-f(t-s)$ is a continuous function of $t \in \mathbb{R}$. As $\tilde{A}$ is closed operator, one obtains that

$$
\begin{gathered}
u_{\varepsilon}(t)=\int_{\mathbb{R}} u(t-s) \alpha_{\varepsilon}(s) d s \in \mathscr{D}(\tilde{A}), \\
\tilde{A} \int_{\mathbb{R}} u(t-s) \alpha_{\varepsilon}(s) d s=\int_{\mathbb{R}}(\tilde{A} u)(t-s) \alpha_{\varepsilon}(s) d s .
\end{gathered}
$$


Now we have

$$
u_{\varepsilon}^{\prime \prime}(t)=\int_{\mathbb{R}} u^{\prime \prime}(t-s) \alpha_{\varepsilon}(s) d s=\int_{\mathbb{R}}[(\tilde{A} u)(t-s)+f(t-s)] \alpha_{\varepsilon}(s) d s,
$$

that is also

$$
\begin{aligned}
u_{\varepsilon}^{\prime \prime}(t) & =\int_{\mathbb{R}}(\tilde{A} u)(t-s) \alpha_{\varepsilon}(s) d s+\int_{\mathbb{R}} f(t-s) \alpha_{\varepsilon}(s) d s \\
& =\tilde{A} u_{\varepsilon}(t)+f \varepsilon(t), \quad \forall t \in \mathbb{R} .
\end{aligned}
$$

On the other hand, we see that the first derivative $u_{\varepsilon}^{\prime}(t)$ is given by

$$
u_{\varepsilon}^{\prime}(t)=\int_{\mathbb{R}} u(\tau) \alpha_{\varepsilon}^{\prime}(t-\tau) d \tau,
$$

so that we estimate $u_{\varepsilon}^{\prime}(\cdot)$ over $\mathbb{R}$, to get

$$
\left\|u_{\varepsilon}^{\prime}(t)\right\| \leq \sup _{\mathbb{R}}\|u(\cdot)\| \int_{\mathbb{R}}\left|\alpha_{\varepsilon}^{\prime}(\sigma)\right| d \sigma=c_{\varepsilon} \sup _{\mathbb{R}}\|u(\cdot)\|, \quad \forall t \in \mathbb{R} .
$$

Thus, for all $\varepsilon>0, u_{\varepsilon}^{\prime}(t)$ is bounded over $\mathbb{R}$.

As in (3.2), (3.3), and (3.5) -applied to $u_{\varepsilon}(\cdot)$-one obtains that

$$
\hat{u}_{\varepsilon}(\lambda) \in \mathscr{D}(\tilde{A}), \quad \forall \lambda \in \mathbb{R}, \quad\left(\lambda^{2}+\tilde{A}\right) \hat{u}_{\varepsilon}(\lambda)=-\hat{f}_{\varepsilon}(\lambda), \quad \forall \lambda \in \mathbb{R} .
$$

Now, using [1, Lemma, page 72], one infers that

$$
\hat{u}_{\varepsilon}(\lambda)=\hat{u}(\lambda) \int_{\mathbb{R}} e^{-i \lambda \sigma} \alpha_{\varepsilon}(\sigma) d \sigma, \quad \hat{f}_{\varepsilon}(\lambda)=\hat{f}(\lambda) \int_{\mathbb{R}} e^{-i \lambda \sigma} \alpha_{\varepsilon}(\sigma) d \sigma,
$$

so that

$$
\begin{gathered}
\lambda^{2} \hat{u}(\lambda) \int_{\mathbb{R}} e^{-i \lambda \sigma} \alpha_{\varepsilon}(\sigma) d \sigma+\tilde{A}\left[\int_{\mathbb{R}} e^{-i \lambda \sigma} \alpha_{\varepsilon}(\sigma) d \sigma\right] \hat{u}(\lambda) \\
=-\hat{f}(\lambda) \int_{\mathbb{R}} e^{-i \lambda \sigma} \alpha_{\varepsilon}(\sigma) d \sigma, \quad \forall \lambda \in \mathbb{R} .
\end{gathered}
$$

Now consider a sequence $\alpha_{p}(\cdot)$ in $C_{0}^{2}(\mathbb{R}), \alpha_{p}(t)=p \alpha(p t)$, for all $p \in \mathbb{N}, \alpha(\cdot) \in$ $C_{0}^{2}(\mathbb{R}),=0$ for $|t| \geq 1, \geq 0$ for all $t \in \mathbb{R}, \int_{\mathbb{R}} \alpha(\cdot) d \sigma=1$. Consider (3.43) for $\alpha_{p}(\sigma)$, and note that $\int_{\mathbb{R}} e^{-i \lambda \sigma} \alpha_{p}(\sigma) d \sigma \rightarrow 1$ as $p \rightarrow \infty$, for all $\lambda \in \mathbb{R}$. We also have

$$
\begin{gathered}
\hat{u}(\lambda) \int_{\mathbb{R}} e^{-i \lambda \sigma} \alpha_{p}(\sigma) d \sigma \rightarrow \hat{u}(\lambda), \quad \forall \lambda \in \mathbb{R}, \\
\hat{A}\left[\hat{u}(\lambda) \int_{\mathbb{R}} e^{-i \lambda \sigma} \alpha_{p}(\sigma) d \sigma\right] \rightarrow-\hat{f}(\lambda)-\lambda^{2} \hat{u}(\lambda), \quad \forall \lambda \in \mathbb{R} .
\end{gathered}
$$

This shows that $\hat{u}(\lambda) \in \mathscr{D}(\tilde{A})$ and $\tilde{A} \hat{u}(\lambda)=-\hat{f}(\lambda)-\lambda^{2} \hat{u}(\lambda)$, for all $\lambda \in \mathbb{R}$.

\section{REFERENCES}

[1] L. Amerio and G. Prouse, Almost-Periodic Functions and Functional Equations, Van Nostrand Reinhold, New York, 1971. MR 43\#819. Zbl 215.15701.

[2] J. L. Dalec'kiĭ and M. G. Kreĭn, Stability of Solutions of Differential Equations in Banach Space, Translations of Mathematical Monographs, vol. 43, American Mathematical Society, Rhode Island, 1974. MR 50\#5126. Zbl 0286.34094. 
[3] H. O. Fattorini, The Cauchy Problem, Encyclopedia of Mathematics and its Applications, vol. 18, Addison-Wesley, Massachusetts, 1983. MR 84g:34003.

[4] C. Lizama, Mild almost periodic solutions of abstract differential equations, J. Math. Anal. Appl. 143 (1989), no. 2, 560-571. MR 91c:34064. Zbl 698.47035.

[5] J. Prüss, On the spectrum of $C_{0}$-semigroups, Trans. Amer. Math. Soc. 284 (1984), no. 2, 847-857. MR 85f:47044.

[6] A. S. Rao and W. Hengartner, On the spectrum of almost periodic solutions of an $a b$ stract differential equation, J. Austral. Math. Soc. 18 (1974), 385-387. MR 51\#3637. Zbl 294.34034.

[7] S. Zaidman, Spectrum of almost-periodic solutions for some abstract differential equations, J. Math. Anal. Appl. 28 (1969), 336-338. MR 39\#7303. Zbl 187.08901.

[8] _ Almost-Periodic Functions in Abstract Spaces, Research Notes in Mathematics, vol. 126, Pitman, Massachusetts, 1985. MR 86j:42018. Zbl 648.42006.

[9]__ Topics in Abstract Differential Equations, Pitman Research Notes in Mathematics Series, vol. 304, Longman Scientific \& Technical, Harlow, 1994. MR 95f:34087. Zbl 806.34001.

[10] _ Topics in Abstract Differential Equations. II, Pitman Research Notes in Mathematics Series, vol. 321, Longman Scientific \& Technical, Harlow, 1995. MR 96f:34081. Zbl 834.34001.

[11] _ Functional Analysis and Differential Equations in Abstract Spaces, Chapman \& Hall/CRC Monographs and Surveys in Pure and Applied Mathematics, vol. 100, Chapman \& Hall/CRC, Florida, 1999. MR 2000e:34103. Zbl 937.47049.

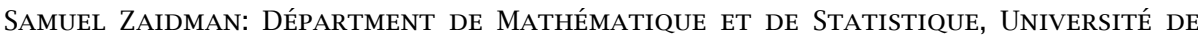
MONTRÉAL, MONTRÉAL, QC, CANADA H3C 3J7

E-mail address: zaidman@dms . umontrea1.ca 


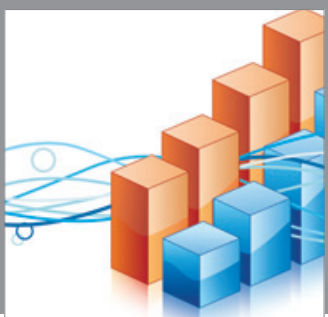

Advances in

Operations Research

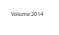

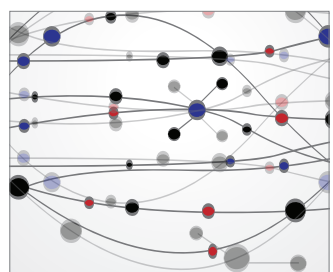

\section{The Scientific} World Journal
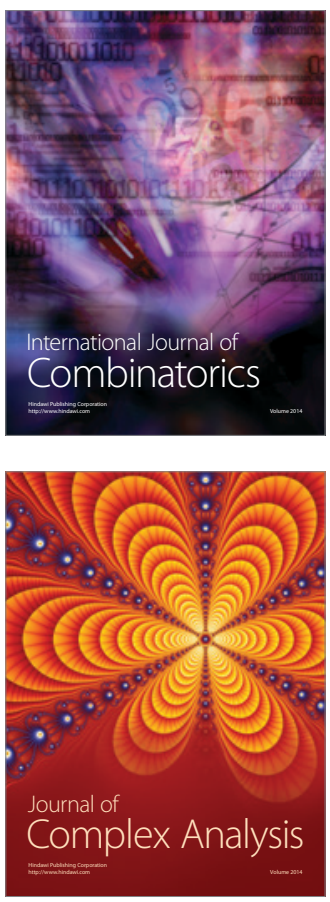

International Journal of

Mathematics and

Mathematical

Sciences
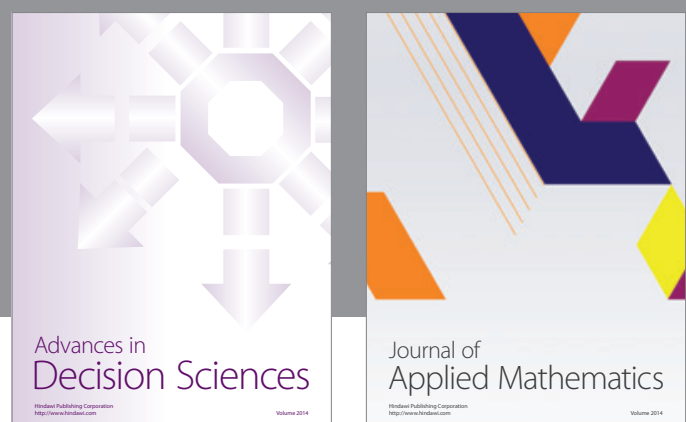

Journal of

Applied Mathematics
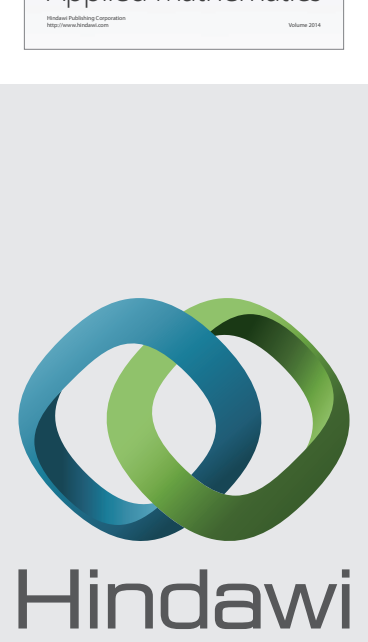

Submit your manuscripts at http://www.hindawi.com
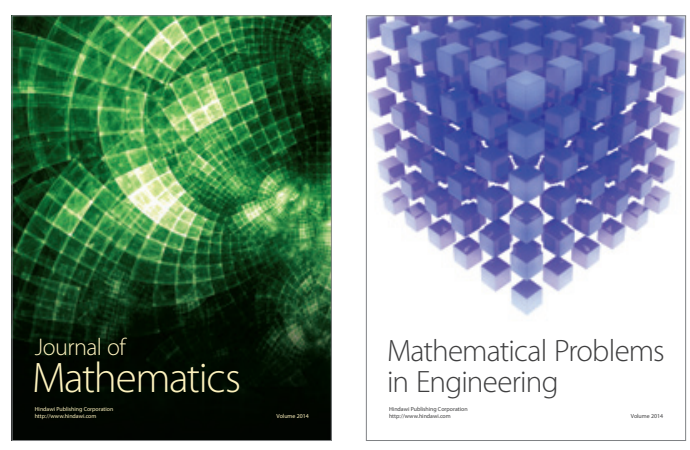

Mathematical Problems in Engineering
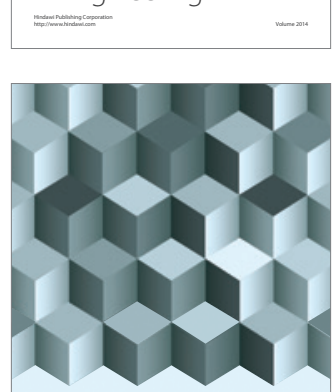

Journal of

Function Spaces
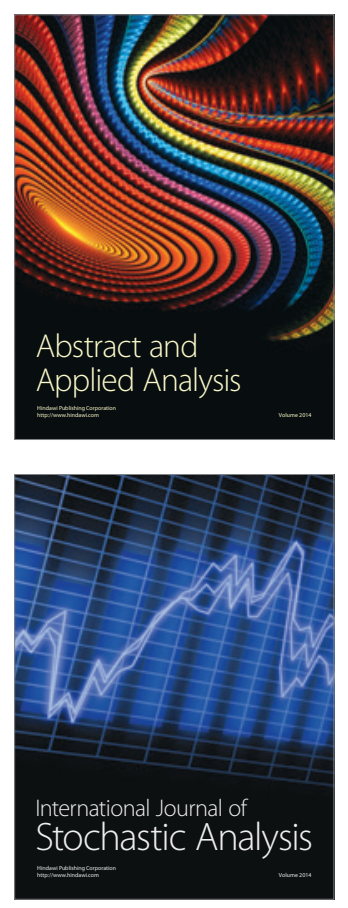

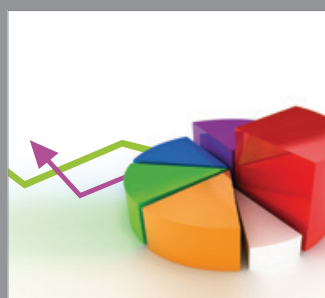

ournal of

Probability and Statistics

Promensencen
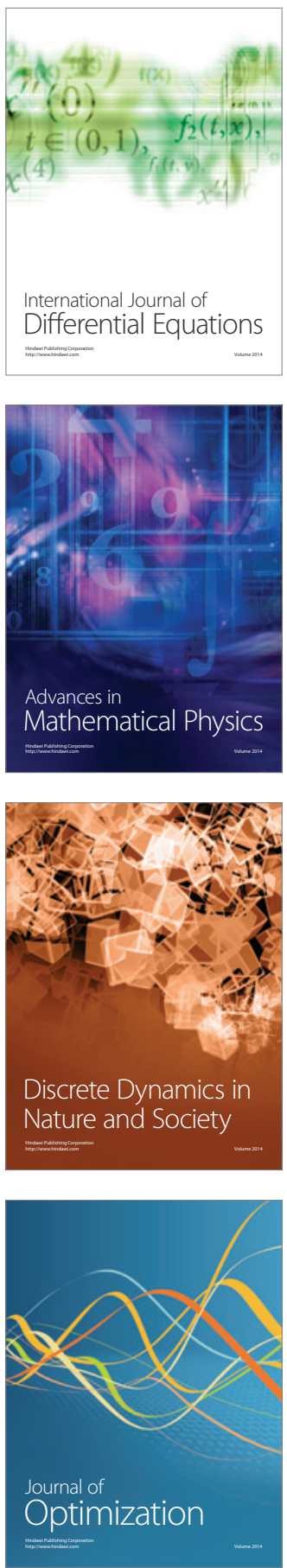